\title{
Novel treatment options in depression and psychosis
}

This article was published in the following Dove Press journal:

Neuropsychiatric Disease and Treatment

\section{Eva Ceskova \\ Petr Silhan}

Department of Psychiatry, University Hospital Ostrava, Ostrava, Czech Republic
Correspondence: Eva Ceskova Department of Psychiatry, Faculty Hospital Brno, Jihlavska 20, 62500

Brno - Bohunice, Czech Republic

Tel +420 532232569

Fax +420 532233706

Email eva.ceskova@gmail.com
Abstract: In spite of tremendous development in central nervous system research, current treatment is suboptimal, especially in severe mental disorders. In medicine, there are two main methods of improving the health care provided: seeking new treatment procedures and perfecting (optimizing) the existing ones. Optimization of treatment includes not only practical tools such as therapeutic drug monitoring but also implementation of general trends in the clinical practice. New pharmacological options include new more sophisticated forms of monoaminergic drugs, old drugs rediscovered on the base of a better understanding of pathophysiology of mental illnesses, and drugs aimed at new treatment targets. In depression, treatment resistance to antidepressive pharmacotherapy represents one of the most important clinical challenges. Switching to monotherapy with new multimodal/multifunctional antidepressants and augmentation with new atypical antipsychotics (aripiprazole and brexpiprazole) may be promising options. Further, current evidence supports utility and safety of adjunctive treatment of nutraceuticals. Novel approaches being studied include ketamine and opioids. Recent advances in technology and emerging knowledge about dysfunctional brain circuits and neuroplasticity have led to the development of different new neuromodulation techniques usually used as add-on therapy. Antipsychotics are still the cornerstone of the current treatment of schizophrenia. Two new partial dopamine agonists, brexpiprazole and cariprazine, are now available in addition to aripiprazole. Although the mechanisms of action are similar, the two agents differ in terms of their pharmacodynamic profiles. Further, two new formulations of long-acting injections of second-generation antipsychotics (aripiprazole lauroxil and 3-month paliperidone palmitate) were introduced into clinical practice. New treatment options not yet available include cannabidiol, glutamate modulators, and nicotine receptors agonists.

Keywords: optimization of treatment, multimodal/multifunctional antidepressants, partial dopamine agonists, glutamate modulators, nicotine receptors agonists

\section{Introduction (burden of mental disease)}

Mental disorders are a major public health concern. The global lifetime prevalence of mental disorders in adults is estimated to be between $12.2 \%$ and $48.6 \%{ }^{1}$ Mental disorders are also a leading contributor to the global disease burden: in 2013, 5.4\% of global disability-adjusted life years and $17.4 \%$ of global years lived with disability were due to mental disorders. ${ }^{2}$

However, current treatment is suboptimal, especially in severe mental disorders such as depressive disorder and schizophrenia. There are two main methods of improving the health care provided: seeking new treatment procedures and perfecting (optimizing) the existing ones. 


\section{Optimizing the treatment in psychiatry}

In all of medicine there is a general consensus, encoded in treatment guidelines, that treatment should be evidencebased, measurement-based, complex, and individualized (personalized).

However, the introduction of these principles in clinical practice is not at all satisfactory. This fact calls for better education of medical students, physicians in primary care, specialists, and all prescribers, and for improvement of the current treatment.

Measurement-based care, increasingly recognized as a treatment enhancement, has become more common with availability of paper or computerized questionnaires and structured interviews. It provides opportunities to increase precision, consistency, and appropriateness of care. However, clinicians are still reluctant to incorporate measurement of outcomes into their practices. The reasons for this resistance are manifold.

Despite the clear and positive evidence for the use of antipsychotics and antidepressants (ADs) that have informed corresponding treatment guidelines, there are substantial reasons to search for ways to improve treatment effectiveness. Emerging evidence indicates that treatment context profoundly affects psychopharmacological interventions. This supports the idea of complex treatment, which includes not only pharmacotherapy but also psychosocial approaches. Pharmacological approaches should always be combined with nonpharmacological approaches, optimizing environmental influences. The influence of psychotropic drugs on brain functions is in continuous interaction with brain plasticity stimulated by environmental influences. The positive contextual factors such as support of positive social interactions and regular physical activities should be introduced into clinical practice. ${ }^{3}$

Clinicians tailor (personalize) treatment depending on particular clinical features, the individual psychopathology, individual risk profile, and patient's experience and preference, even in the absence of strong evidence that such features are truly predictive. Further, patients differ in their ability to absorb, distribute, metabolize, and excrete drugs due to concurrent disease, age, concomitant medication, or genetic peculiarities. The importance of sex-related pharmacologic differences in drug pharmacokinetics and pharmacodynamics is now indisputable. ${ }^{4}$ To overcome problems of nonresponse or adverse reactions due to pharmacokinetic abnormalities, it is helpful to monitor the pharmacokinetic characteristics of individual patients and optimize the drug concentration.
The analysis and interpretation of plasma levels (therapeutic drug monitoring, TDM) is available; however, this method has not yet been used routinely and it seems unlikely that TDM will become a standard of care for all agents and all patients. Published guidelines for TDM indicate for which patients and what circumstances TDM is cost-effective. Further, TDM presents an adherence-improving strategy that has a tremendous potential for reducing health care costs, personal suffering, and the burden on families. ${ }^{5}$ Low adherence is a major problem of long-term psychotropic treatment. Insufficient adherence in psychiatry, especially in psychotic patients $(\sim 50 \%)$, has severe consequences. Its prevalence has not changed significantly with the introduction of new medications. ${ }^{6}$

Aiming for more precise treatment, individualization may help to ensure optimal outcomes. ${ }^{7}$ To achieve true precision in pharmacotherapy, it may be necessary to identify and deploy treatment-specific predictors of response. Combinatorial pharmacogenomic testing encompassing more complete genomic information by combining moderate-risk alleles has become commercially available. ${ }^{8}$

Patient-centered medicine seeks to focus attention first on the need and concerns of the patient and to consider social and economic factors. ${ }^{9}$ Knowledge of the pharmacology of psychiatric medications is necessary but not sufficient for the delivery of appropriate, safe, and effective care. The clinician needs to devote adequate time for communicating treatment needs to patients and develop a strong treatment alliance that persists even during symptomatic exacerbations..$^{10}$

\section{Depression}

Major depressive disorder (MDD) is a widespread disease, with a lifetime prevalence of $15 \%$ and an annual incidence of $\sim 7 \%$. It is associated with significant costs in quality of life, loss of work productivity, and a high risk of mortality. ${ }^{11}$

About two-thirds of patients do not achieve full remission, which is key to restoring full functioning and preventing relapse. After excluding pseudoresistance, several treatment strategies are available for these patients, such as changing, augmenting, and combining ADs.

\section{New treatment options currently available \\ Multimodal ADs}

Multimodal ADs have more than one mode of action, ie, reuptake inhibition and activity on membrane-bound receptors, pooling two distinct modes of neural signaling. Through 
these multimodal mechanisms, they produce a downstream effect on interconnected neurotransmitter systems. The differences among available multimodal/multifunctional ADs are based on their action at receptor subtypes. Against this background, we feature the new (the last registered) ADs vortioxetine (Trintellix) and vilazodone (Viibryd) with regard to their serotonin receptor targets $5-\mathrm{HT}_{1 \mathrm{~A}}, 5-\mathrm{HT}_{3}$, and $5-\mathrm{HT}_{7}$, which may account for their specific effects on certain symptoms of depression (eg, cognition and anxiety) as well as a characteristic side effect profile. The efficacy of new multimodal ADs has not yet been tested in treatmentresistant depression. ${ }^{12,13}$

Drugs targeting the glutamatergic system might open up a promising new territory for the development of drugs to meet the needs of patients with major depression.

\section{Ketamine}

Ketamine is an anesthetic drug with N-methyl-D-aspartate (NMDA) glutamate receptor antagonist activity, which may provide a novel AD mechanism. Ketamine has several brand names for the drugs in which it is the sole medication. According to a recently published systematic search for relevant randomized trials, ketamine does indeed have a rapid but transient AD effect. However, there is substantial heterogeneity in clinical response, and ketamine's underlying mechanisms of action are not entirely understood. ${ }^{14,15}$

The observation that a single intravenous dose of glutamatergic modulator ketamine produces a robust and rapid $\mathrm{AD}$ effect was a turning point that opened the way for other, more selective glutamatergic modulators (intranasal esketamine, rapastinel). ${ }^{16}$

\section{New drugs Opioids}

The AD potential of opioids has been known for centuries, but their dependence-producing properties led to the rapid adoption of monoamine-based ADs once they become available in the 1950s. Nowadays, a growing body of preclinical and clinical evidence supporting the hypothesis that mood disorders involve deregulation of the endogenous opioid system is emerging. The contemporary clinical use of opioid agonists as ADs in clinical practice remains highly limited because of unresolved issues of abuse and dependence. ${ }^{17}$ To overcome the limitations of opioid agonists, a combination of a $\mu$ and $\kappa$ opioid partial agonist, buprenorphine, and a mu opioid antagonist samidorphan were developed. The results of a multicenter, randomized, double-blind, placebo-controlled study have shown that the modulation of the opiate system may be a novel treatment approach for treatment-resistant depression. ${ }^{18}$

\section{Psychedelics}

The psychedelic drugs, including lysergic acid diethylamide and psilocybin, were used extensively in the treatment of mood disorders and other psychiatric conditions, before their prohibition in the late $1960 \mathrm{~s} .{ }^{19}$ A recently published systematic review of clinical treatment studies using psychedelics in patients with broadly defined depression included 423 individuals in 19 studies, and 335 (79.2\%) showed clinicianjudged improvement after treatment with psychedelics. ${ }^{20}$ A recently completed pilot study in the UK favors the use of psilocybin with psychological support in treatment-resistant depressive disorder. Psychedelics may exert therapeutic effects for psychiatric disorders by acutely destabilizing local brain network hubs and global network connectivity, providing the occasion for brain network "resetting" after the acute effects have resolved. ${ }^{21}$

\section{New augmentation strategy}

The most evidence-based augmentation of specific serotonin reuptake inhibitors is an augmentation with atypical antipsychotics. Recently, the spectrum of atypical antipsychotics used in this indication has been enlarged by brexpiprazole (Rexulti, approved by the US Food and Drug Administration in 2015), a new partial dopamine agonist. ${ }^{22}$

Recently, several reviews showed evidence of efficacy of a safe augmentation strategy - nutraceuticals. The coadministration of standardized pharmaceutical-grade nutrients, referred to as nutraceuticals, may provide an effective and safe approach to enhancing AD effects, by either synergistically augmenting a particular activity of an AD or providing a range of additional biological effects. ${ }^{23}$ All nutraceuticals are generally well tolerated, with gastrointestinal adverse events most commonly reported across all nutraceutical groups. Current evidence supports adjunctive use of S-adenosylmethionine, methylfolate, omega-3, and vitamin D with ADs to reduce depressive symptoms. ${ }^{23}$

However, depression is not simply the result of neurotransmitter abnormalities. A large body of evidence points to a deregulated endocrine and inflammatory response system and reduced neurogenesis, in the pathogenesis of depression. MDD is associated with a deregulation of immune response and with chronic inflammatory activity as reflected by abnormal profiles of circulating pro- and anti-inflammatory cytokines. Several studies indicate that the adjunctive use of anti-inflammatory agents in patients with MDD improves 
depressive symptoms, in particular in treatment-resistant depression with an inflammatory profile as defined by a C-reactive protein. ${ }^{24}$ Several anti-Il-1 $\beta$-targeted compounds have been recently developed, including antibodies and Il-1 $\beta$ receptor antagonist. Targeting interleukin- $1 \beta$ may be useful for subgroups of patients with inflammatory depression that is defined by clinical features of trauma, resistance to $\mathrm{AD}$, and raised interleukin-1 levels. ${ }^{25}$

Further, advances in technology and emerging knowledge about the dysfunctional brain circuits underlying depression and neuroplasticity have led to the development of different new neuromodulation techniques (transcranial magnetic stimulation, vagus nerve stimulation, and deep brain stimulation) usually used as add-on therapy. ${ }^{26}$

\section{Schizophrenia}

Schizophrenia, a chronic brain disorder that affects $\sim 1 \%$ of the adult population and $\sim 26$ million people worldwide, is considered among the most disabling and economically catastrophic medical disorders as ranked by the World Health Organization. ${ }^{27}$ Only $\sim 10 \%-15 \%$ of people who suffer from schizophrenia maintain full-time employment of any type. ${ }^{28}$

Because the etiology of schizophrenia is unknown, the goals of treatment are to eliminate disease-related symptoms and to enhance functioning. The cornerstone of the current treatment is antipsychotic medication. Antipsychotics vary in terms of tolerability and safety concerns, and patients themselves differ in terms of preexisting risk factors and comorbidities that make drug selection often challenging.

\section{New treatment options currently available Antipsychotics}

In addition to aripiprazole, two new dopamine receptor partial agonists, brexpiprazole and cariprazine (Vraylar), are now available. Although the mechanisms of action are similar, the three agents differ in terms of their pharmacodynamic profiles. ${ }^{29}$ Compared with aripiprazole, brexpiprazole has low intrinsic activity at the dopamine $\mathrm{D}_{2}$ receptor and has an approximately tenfold higher affinity for serotonin 5-HT and $5-\mathrm{HT}_{2 \mathrm{~A}}$ receptors, potentially enhancing tolerability. When cariprazine was compared with aripiprazole, similar $\mathrm{D}_{2}$ and three- to tenfold greater $\mathrm{D}_{3}$ versus $\mathrm{D}_{2}$ selectivity was observed for cariprazine. It remains unknown whether targeting the dopamine $\mathrm{D}_{3}$ receptor over the dopamine $\mathrm{D}_{2}$ receptor is clinically advantageous, but theoretically dopamine $\mathrm{D}_{3}$ preferring agents may exert procognitive effects.
Although all three medications are approved for the treatment of schizophrenia, both aripiprazole and brexpiprazole are also approved for adjunctive treatment of MDD, and both aripiprazole and cariprazine are also approved for acute treatment of manic or mixed episodes associated with bipolar I disorder.

In Phase III of clinical development is a novel antipsychotic lumateperone with a unique pharmacological profile. Lumateperone, combining potent $5-\mathrm{HT}_{2 \mathrm{~A}}$ receptor antagonism with cell-type-specific dopamine and glutamate receptor modulation plus serotonin reuptake inhibition, is a novel modulator of serotonin, dopamine, and glutamate neurotransmission. ${ }^{30}$

\section{New formulations of antipsychotics}

Long-acting injections (LAIs) of the second-generation antipsychotics provide continuous therapy facilitating patient participation in treatment and enhancing their recovery. Although LAIs may improve treatment adherence, only a minority of medication-nonadherent patients receive them. Significant reductions in health care utilization or costs associated with schizophrenia have been demonstrated in some studies of LAI antipsychotics, although other studies have not demonstrated these effects and showed cost-neutrality or even greater cost usually in context with the type of study (eg, observations of naturalistic studies contrary to randomized controlled studies). ${ }^{31,32}$

Recently, two new LAI formulations of second-generation antipsychotics have become available, aripiprazole lauroxil (Aristada) and 3-month paliperidone palmitate (Invega Sustenna and Invega Trinza). In October, 2015, the US Food and Drug Administration approved aripiprazole lauroxil, a prodrug of aripiprazole, for the treatment of adults with schizophrenia. Enzyme-mediated hydrolysis likely converts the injected aripiprazole lauroxil to N-hydroxymethyl aripiprazole, which is then hydrolyzed to aripiprazole. Aripiprazole lauroxil works primarily as a result of the activity and affinities for the $\mathrm{D}_{2}$ receptors of the parent drug, aripiprazole, and, to a lesser extent, of its major metabolite, dehydroaripiprazole, which also represents $30 \%-40 \%$ of the aripiprazole exposure in plasma. Aripiprazole lauroxil is metabolized extensively in the liver via cytochrome P450 CYP isoenzymes 3A4 and 2D6. Its pharmacokinetic profile led to approval of dosing intervals of every 6 weeks for the $882 \mathrm{mg}$ dose. The overall tolerability is consistent with what is known about oral aripiprazole. ${ }^{33}$

A new formulation of paliperidone palmitate, paliperidone palmitate 3-month formulation that was recently approved in 
the US for the maintenance treatment of schizophrenia, offers a substantially longer dosing interval of once every 3 months than is available for typical or new-generation atypical LAI formulations (1 monthly). No clinically relevant differences were observed in pharmacokinetic exposures between 3-month and 1-month formulations. Both have similar tolerability profiles and no new safety issues were detected. The new paliperidone LAI with its 3-month dosing interval is a unique option for relapse prevention in schizophrenia. ${ }^{34}$

LAI antipsychotics eliminate the need for daily dosing, typically ensure sustained plasma levels for several weeks, and help to reliably monitor adherence. The new LAI options provide additional flexibility in terms of increasing the time between injections.

\section{New treatment options not yet available in clinical practice Cannabidiol (CBD)}

The endocannabinoid system is a highly promising new pharmacological target in the context of schizophrenia. Modulation of this system by the main psychoactive component in cannabis, $\Delta 9$-tetrahydrocannabinol, induces acute psychotic effects and cognitive impairment. However, the nonpsychotropic, plant-derived cannabinoid agent CBD may have antipsychotic properties and thus may be a promising new agent in the treatment of schizophrenia. The first smallscale clinical studies with CBD for the treatment of patients with psychotic symptoms further confirmed the potential of CBD as an effective, safe, and well-tolerated antipsychotic compound, although large randomized clinical trials will be needed before this novel therapy can be introduced into clinical practice. ${ }^{35}$

\section{Treatment options for negative symptoms and cognitive dysfunction Glutamate modulators}

The glutamatergic system has been implicated in the pathophysiology of schizophrenia and affective disorders. A key component is the dysfunction of the glutamatergic receptor, N-methyl-D-aspartate receptor (NMDAR). Substances regulating activation/inhibition of the NMDAR are promising in therapeutic approaches to negative symptoms, cognition, and mood. ${ }^{36}$ Schizophrenia is related to cognitive and negative symptoms, which often are resistant to current treatment approaches.

The NMDAR hypofunction theory of schizophrenia proposes that enhancement of the receptor function may lead to efficacy against schizophrenia. Consistent with this theory, recent clinical trials have demonstrated that the enhancement of NMDAR function by potentiating the glycine site of the receptor is efficacious in the treatment of negative and possibly cognitive symptoms of schizophrenia. ${ }^{37}$ Metabotropic glutamate receptors (mGluRs) have attracted significant attention as potential drug targets. This is due to the belief that metabotropic receptor targeting provides a way for modulating glutamate tone and phasic release in a subtler manner than that which can be achieved through glutamate ionotropic receptors. Specifically, emerging preclinical and clinical data suggest that activation of Group II mGluRs is a promising approach for the treatment of schizophrenia. Further, metabotropic glutamate receptor subtype 5 (mGluR5), encoded by the GRM5 gene, represents a compelling novel drug target for the treatment of schizophrenia. Accordingly, mGluR5 positive allosteric modulators show encouraging therapeutic potential in preclinical schizophrenia models, particularly for the treatment of cognitive dysfunctions..$^{36,38}$

\section{Nicotine receptors agonists}

The excessive cigarette smoking exhibited by schizophrenic patients suggests that they might be self-medicating to ameliorate certain aspects of the characteristic positive, negative, and cognitive symptoms associated with the disease. Morphological examinations found alterations in nicotinic receptors in postmortem tissue from schizophrenic individuals compared to controls, especially in the $\alpha 7$ nicotinic acetylcholine receptors ( $\alpha 7 \mathrm{nAChRs)} \mathrm{subtypes.} \mathrm{These} \mathrm{data} \mathrm{were} \mathrm{consistent}$ with molecular biology studies which demonstrated associations between polymorphisms in gene coding for these receptors and schizophrenia. The available data show that $\alpha 7 \mathrm{nAChR}$ activation did not improve positive symptoms, whereas a beneficial effect against negative symptoms was observed repeatedly. The clinical evidence that $\alpha 7 \mathrm{nAChR}$ activation leads to improvement of cognitive dysfunction in schizophrenia still remains somewhat equivocal, although the data for encenicline are encouraging. Currently, some other $\alpha 7 \mathrm{nAChR}$ agonists remain in clinical development. ${ }^{39,40}$

\section{Conclusion}

Before developing new psychoactive drugs, it is our duty to optimize the usage of those currently available. Many psychotropic medications are available, but achieving optimal therapeutic response can be challenging. Schizophrenia and MDD are heterogeneous disorders and medication responses (and tolerability) vary across patients. As always, having additional choices can offer greater opportunities for success. With better understanding of pathophysiology, there 
is a revival of new old drugs. In depression, we can see the arrival of the first robust and rapid-acting AD drug in the near future. In schizophrenia, given the challenges with patient adherence to oral regimens and since relapse leads to serious medical and psychosocial consequences, the future of pharmacotherapy is likely to include improved long-term delivery systems. In both depressive and schizophrenic disorders, glutamatergic modulators likely represent a very promising alternative to monoaminergic AD monotherapy.

Advances in genomics and cell biology have increased the opportunity for rational design of targeted drugs. Targeted therapies may offer enhanced efficacy and improved selectivity and therefore less toxicity. As immediate challenge facing the field is to make progress in the development and identification of personalized treatments, perhaps through the application of biomarkers.

\section{Acknowledgment}

The paper was presented at the 13th Congress of the European Association for Clinical Pharmacology and Therapeutics (EACPT) in Prague, Czech Republic, June 24-27, 2017, and the abstract has been published in the EACPT Clinical Therapeutics journal.

\section{Disclosure}

EC has received speaker's honoraria from Angelini Pharma and Jahnssen Cilag, Czech Republic, and PS has received speaker's honoraria from Lundbeck, Czech Republic, and Servier, Czech Republic. The authors report no other conflicts of interest in this work.

\section{References}

1. Demyttenaere K, Bruffaerts R, Posada-Villa J, et al. Prevalence, severity, and unmet need for treatment of mental disorders in the World Health Organization World Mental Health Surveys. JAMA. 2004;291: 2581-2590.

2. Global, regional, and national incidence, prevalence, and years lived with disability for 310 diseases and injuries, 1990-2015: a systematic analysis for the Global Burden of Disease Study 2015. Lancet. 2016; 388:1545-1602.

3. Rief W, Barsky AJ, Bingel U, et al. Rethinking psychopharmacotherapy: the role of treatment context and brain plasticity in antidepressant and antipsychotic interventions. Neurosci Biobehav Rev. 2016;60:51-64.

4. Alyson J, McGregor AJ. The effects of sex and gender on pharmacologic toxicity: implications for clinical therapy. Clin Ther. 2017;39:8-9.

5. Hiemke C, Baumann P, Bergemann N, et al. AGNP consensus guidelines for therapeutic drug monitoring in psychiatry: update 2011. Pharmacopsychiatry. 2011;44:195-235.

6. Lingam R, Scott J. Treatment non-adherence in affective disorders. Acta Psychiatr Scand. 2002;105:164-172.

7. Perlis RH. Abandoning personalization to get to precision in the pharmacotherapy of depression. Word Psychiatry. 2016;15:228-235.

8. El-Mallakh RS, Roberts RJ, El-Mallakh PL, Findlay LJ, Reynolds KK. Pharmacogenomics in psychiatric practice. Clin Lab Med. 2016;36: 507-523.
9. Nichols DE, Bardes CL. Defining "patient-centred medicine". N Eng J Med. 2012;366:782-783.

10. Glick ID, Ellison JM. Improving the practice of clinical psychopharmacotherapy: the process of long-term management for patients and caregivers. J Clin Psychiatry. 2015;76:735-736.

11. Mathers CD, Loncar D. Projections of global mortality and burden of disease from 2002 to 2030. PLoS Med. 2006;3:e442.

12. Ceskova E. Current pharmacotherapy of depression - focused on multimodal/multifunctional antidepressants. Expert Opin Pharmacother. 2016;17:1835-1837.

13. Frampton JE. Vortioxetine: a review in cognitive dysfunction in depression. Drugs. 2016;76:1675-1682.

14. Xu Y, Hackett M, Carter G, et al. Effect of low-dose and very low-dose ketamine among patients with major depression: a systematic review and meta-analysis. Int $J$ Neuropsychopharmacol. 2016;19:1-15.

15. Zhang MW, Ho R. Critical appraisal of existing ketamine trials: existing limitations and limited applicability of treatment. Am J Psychiatry. 2016;173:431.

16. Garay RP, Zarate CA Jr, Charpeaud T, et al. Investigational drugs in recent clinical trials for treatment-resistant depression. Expert Rev Neurother. 2017;29:1-17.

17. Rosenthal RN, Ling W, Casadonte P, et al. Buprenorphine implants for treatment of opioid dependence: randomized comparison to placebo and sublingual buprenorphine/naloxone. Addiction. 2013;108:2141-2149.

18. Fava M, Memisoglu A, Thase ME, et al. Opioid modulation with buprenorphine/samidorphan as adjunctive treatment for inadequate response to antidepressants: a randomized double-blind placebocontrolled trial. Am J Psychiatry. 2016;173:499-508.

19. Patra S. Return of the psychedelics: psilocybin for treatment resistant depression. Asian J Psychiatr. 2016;24:51-52.

20. Rucker JJ, Jelen LA, Flynn S, Frowde KD, Young AH. Psychedelics in the treatment of unipolar mood disorders: a systematic review. J Psychopharmacol. 2016;30:1220-1229.

21. Nichols DE, Johnson MW, Nichols CD. Psychedelics as medicines: an emerging new paradigm. Clin Pharmacol Ther. 2017;101:209-219.

22. Citrome L. Brexpiprazole for schizophrenia and as adjunct for major depressive disorder: a systematic review of the efficacy and safety profile for the newly approved antipsychotic-what is the number needed to treat, number needed to harm and likelihood to be helped or harmed? Int J Clin Pract. 2015;69:978-997.

23. Sarris J, Murphy J, Mischoulon D, et al. Adjunctive nutraceuticals for depression: a systematic review and meta-analyses. Am J Psychiatry. 2016;173:575-587.

24. Raison CL, Rutherford RE, Woolwine BJ, et al. A randomized controlled trial of the tumor necrosis factor antagonist infliximab for treatmentresistant depression: the role of baseline inflammatory biomarkers. JAMA Psychiatry. 2013;70:31-41.

25. Ellul P, Boyer L, Groc L, Leboyer M, Fond G. Interleukin-1beta-targeted treatment strategies in inflammatory depression: toward personalized care. Acta Psych Scand. 2016;134:469-484.

26. Bewernick B, Schlaepfer TE. Update on neuromodulation for treatmentresistant depression. Version 1. F1000Res. 2015;4:F1000 Faculty Rev-1389.

27. Murray CJ, Lopez AD. Evidence-based health policy-lessons from the Global Burden of Disease Study. Science. 1996;274:740-743.

28. Cloutier M, Aigbogun MS, Guerin A, et al. The economic burden of schizophrenia in the United States in 2013. J Clin Psychiatry. 2016; 77:764-771.

29. Citrome L. The ABC's of dopamine receptor partial agonists-aripiprazole, brexpiprazole and cariprazine: the 15-min challenge to sort these agents out. Int J Clin Practice. 2015;69:1211-1220.

30. Davis RE, Correll CU. ITI-007 in the treatment of schizophrenia: from novel pharmacology to clinical outcomes. Expert Rev Neurother. 2016;16:601-614.

31. Kishimoto T, Nitta M, Borenstein M, Kane JM, Correll CU. Long-acting injectable versus oral antipsychotics in schizophrenia: a systematic review and meta-analysis of mirror-image studies. J Clin Psychiatr. 2013;74:957-965. 
32. Kishimoto T, Robenzadeh A, Leucht C, et al. Long-acting injectable vs oral antipsychotics for relapse prevention in schizophrenia: a metaanalysis of randomized trials. Schizophr Bull. 2014;40:192-213.

33. Cruz MP. Aripiprazole lauroxil (Aristada). An extended-release, long-acting injection for the treatment of schizophrenia. P T. 2016;41: 556-559.

34. Savitz AJ, Xu H, Gopal S, et al. Efficacy and safety of paliperidone palmitate 3-month formulation for patients with schizophrenia: a randomized, multicenter, double-blind, noninferiority study. Int $J$ Neuropsychopharmacol. 2016;19:pyw018.

35. Leweke FM, Mueller JK, Lange B, Rohleder C. Therapeutic potential of cannabinoids in psychosis. Biol Psychiatry. 2016;79:604-612.

36. Henter ID, de Sousa RT, Gold PW, Brunoni AR, Zarate CA, MachadoVieira R. Mood therapeutics: novel approaches for treating depression. Expert Rev Clin Pharmacol. 2017;10:153-166.
37. Shim SS, Hammonds MD, Kee BS. Potentiation of the NMDA receptor in the treatment of schizophrenia: focused on the glycine site. Eur Arch Psychiatry Clin Neurosci. 2008;258:16-27.

38. Hashimoto K, Malchow B, Falkai P, Schmitt A. Glutamate modulators as potential therapeutic drugs in schizophrenia and affective disorders. Eur Arch Psychiatry Clin Neurosci. 2013;263:367-377.

39. Adams CE, Stevens KE. Evidence for a role of nicotinic acetylcholine receptors in schizophrenia. Front Biosci. 2007;12:4755-4772.

40. Kalkman HO, Feuerbach D. Modulatory effects of $\alpha 7 \mathrm{nAChRs}$ on the immune system and its relevance for CNS disorders. Cell Mol Life Sci. 2016;73:2511-2530.
Neuropsychiatric Disease and Treatment

\section{Publish your work in this journal}

Neuropsychiatric Disease and Treatment is an international, peerreviewed journal of clinical therapeutics and pharmacology focusing on concise rapid reporting of clinical or pre-clinical studies on a range of neuropsychiatric and neurological disorders. This journal is indexed on PubMed Central, the 'PsycINFO' database and CAS,

\section{Dovepress}

and is the official journal of The International Neuropsychiatric Association (INA). The manuscript management system is completely online and includes a very quick and fair peer-review system, which is all easy to use. Visit http://www.dovepress.com/testimonials.php to read real quotes from published authors.

Submit your manuscript here: http://www.dovepress.com/neuropsychiatric-disease-and-treatment-journal 